Service social

\title{
Familles d'accueil et prévention des MTS et du VIH-Sida auprès de jeunes : épilogue d'un programme à succès
}

\section{Marie Berlinguet et Germain Trottier}

Volume 45, numéro 1, 1996

Le sexuel et le relationnel

URI : https://id.erudit.org/iderudit/706719ar

DOI : https://doi.org/10.7202/706719ar

Aller au sommaire du numéro

Éditeur(s)

École de service social de l'Université Laval

ISSN

1708-1734 (numérique)

Découvrir la revue

Citer cet article

Berlinguet, M. \& Trottier, G. (1996). Familles d'accueil et prévention des MTS et du VIH-Sida auprès de jeunes : épilogue d'un programme à succès. Service social, 45(1), 137-145. https://doi.org/10.7202/706719ar d'utilisation que vous pouvez consulter en ligne. 


\title{
COMMENTARES ET DOCUMENTS
}

\section{Familles d'accueil et prévention des MTS et du VIH-sida auprès de jeunes: épilogue d'un programme à succès}

\author{
Hommage aux parents substituts \\ qui assument au quotidien \\ la responsabilité des enfants des autres
}

MARIE BERLINGUET

Travailleuse sociale, chercheuse associée

GERMAIN TROTTIER

Travailleur social, professeur École de service social, Université Laval

Il est plutôt inhabituel que des chercheurs se permettent de formuler a posteriori des commentaires sur les caractéristiques et les retombées d'un projet déjà évalué et diffusé. Cependant, le succès remporté jusqu'ici par ce programme mérite, à notre avis, qu'on s'y arrête et qu'on en tire des leçons pouvant être utiles à la pratique et à la recherche.

Les auteurs sont membres de l'équipe de recherche sociale subventionnée par le Conseil québécois de la recherche sociale : "La prévention des MTS et du sida : dimensions psychosociales et socioculturelles ». 
Le programme dont il est question a été édité par le ministère de la Santé et des Services sociaux ${ }^{1}$ et a fait l'objet de deux communications scientifiques ${ }^{2}$. Décrit dans les histoires à succès du Forum national sur la santé ${ }^{3}$, il a été l'un des trois projets finalistes au dernier concours de l'Association des Centres jeunesse du Québec dans la catégorie "support à l'intervention clinique $^{4} »$. De plus, en décembre 1996, le Centre québécois de coordination sur le sida (CQCS), conjointement avec le Centre jeunesse de Québec, donnera une session de formation à l'intention de personnes-ressources des Régies régionales de la santé et des services sociaux et des Centres jeunesse du Québec en vue de l'implantation du programme dans les Centres jeunesse du territoire québécois.

Bien que le projet ait été largement diffusé, nous le présentons ici afin d'en discuter les points saillants et de soumettre aux lecteurs les réflexions qui en ont surgi.

\section{DESCRIPTION DU PROGRAMME}

Il s'agit d'un programme de formation intitulé "Parents d'accueil et prévention des MTS et du VIH-sida chez les adolescentes et les adolescents à risque ». Il a été conçu, élaboré et réalisé par un groupe de cinq intervenantes du Centre jeunesse de Québec. Ces intervenantes proviennent de trois disciplines: le service social, l'éducation et les sciences infirmières 5 .

Le programme est basé sur les considérations suivantes:

- L'importance de la prévention des MTS et du VIH-sida, qui représentent des risques majeurs pour la santé. Ainsi, le sida peut entraîner la mort et la chlamydia non traitée peut amener l'infertilité.

- Les jeunes constituent une population particulièrement vulnérable face aux MTS et au VIH-sida et les jeunes en difficulté d'adaptation sociale montrent plus de comportements à risque que les autres adolescents (Godin, 1991).

- Les jeunes expriment le souhait d'entendre parler des relations amoureuses en prévention des MTS et du VIH-sida.

- Les parents d'accueil sont les adultes-ressources les plus en contact direct avec les jeunes en placement; ils sont susceptibles de constituer pour les jeunes des modèles valides d'identification et d'être en position avantageuse pour établir avec eux des relations significatives. 
L'intention du programme est de rendre les parents d'accueil aptes à assumer un rôle de guides en matière de prévention des MTS-sida, auprès des jeunes qui leur sont confiés par le Centre jeunesse. Il faut noter ici que ce programme n'a pas pour but de faire la promotion d'une vie sexuelle active, mais qu'il vise plutôt à promouvoir une sexualité responsable et préventive respectant l'évolution du jeune lui-même.

Plus spécifiquement, le programme poursuit les objectifs suivants auprès des parents d'accueil :

- Améliorer leurs connaissances pour intervenir adéquatement en matière de prévention des MTS-sida dans le contexte des relations amoureuses des jeunes en placement (savoir).

- Susciter la réflexion sur des attitudes favorables à la communication en lien avec la vie amoureuse et sexuelle des jeunes (savoir-être).

- Adopter des comportements et des stratégies susceptibles de renforcer leurs interventions en sexualité préventive, selon les demandes et les besoins des jeunes (savoir-faire).

Le programme prévoit cinq rencontres hebdomadaires consécutives, d'une durée de trois heures chacune, avec un thème central pour chaque rencontre. Les cinq thèmes abordés sont les suivants :

1. On fait connaissance et on se rappelle.

2. La vie amoureuse des jeunes.

3. Les MTS-sida Phase 1: les connaissances.

4. Les MTS-sida Phase 2: les moyens de prévention.

5. La parole est à vous.

L'approche est de type "groupe structuré», comportant la projection de documents audiovisuels, des mises en situation, des exercices d'auto-évaluation de connaissances et d'attitudes, de même que des périodes de discussion.

Les familles d'accueil ont été invitées à s'inscrire sur une base volontaire. Quarante et un participantes et participants représentant trente-deux familles de divers types (familles régulières, de dépannage, de réadaptation et spécifiques) se sont inscrits à l'un ou l'autre des groupes formés dans trois sousrégions desservies par le Centre jeunesse de Québec: Québec, Portneuf et Charlevoix. Soixante et un adolescents et adolescentes (20 garçons et 41 filles) étaient sous leur responsabilité. 


\section{ÉVALUATION DU PROGRAMME}

L'évaluation externe du programme a été faite par un professeurchercheur de l'Université Laval. Quatre indicateurs ont été retenus pour l'évaluation sommative du projet: 1) la constance de participation des parents participants ; 2) leur degré de satisfaction par rapport au programme; 3) l'accroissement des connaissances en matière de prévention des MTS et du sida et 4) l'adoption de stratégies de transmission des connaissances aux adolescents et aux adolescentes. Un mois après la fin du programme, l'évaluateur externe a réuni les animatrices du programme pour obtenir ex post facto leurs perceptions et commentaires sur trois aspects principaux : 1) les efforts exigés pour donner le programme; 2) le degré de satisfaction des intervenantes et 3) les retombées attendues de l'expérience. Cet exercice avait pour but d'évaluer de façon formative le programme réalisé.

Les évaluations sommative et formative du projet mettent en évidence: 1) que les parents de ces familles d'accueil sont intéressés à parler de la vie affective des jeunes et de leur sexualité, et qu'ils sont à l'aise pour le faire ; 2) qu'ils sont motivés et enthousiastes à jouer un rôle d'éducation préventive en matière de MTS et du sida et 3) que le programme leur a convenu et qu'il pourrait être profitable à tous les parents, de même qu'aux jeunes. À noter qu'un programme est actuellement en préparation à l'intention des parents d'accueil et des jeunes qui leur sont confiés.

\section{Points saillants : propos et commentaires}

Nous croyons pertinent de présenter et de commenter ici un certain nombre de caractéristiques du programme, souhaitant que nos réflexions puissent être utiles aux praticiennes, praticiens, chercheuses et chercheurs dans le domaine de la prévention des MTS et du VIH-sida, mais également dans le champ plus général du service social.

\section{Une approche inédite}

L'idée de recourir aux parents d'accueil comme intermédiaires dans la prévention des MTS et du VIH-sida auprès des jeunes représente une innovation dans le domaine. En effet, les promo- 
teurs des programmes de santé publique avaient visé jusqu'ici des groupes cibles, tels les jeunes à risque, les hommes ayant des relations sexuelles avec d'autres hommes, les femmes, les intervenantes et les intervenants en santé, les éducateurs et les éducatrices en milieu scolaire ou dans les centres jeunesse de réadaptation, les animateurs et les animatrices des maisons de jeunes, etc., pour recevoir une formation adéquate relativement à cette problématique. Pourtant, les parents de familles d'accueil n'avaient jamais reçu de formation adaptée à leur rôle de parents substituts. Les parents d'accueil vivent des conflits intergénérationnels comme tous les autres parents par rapport au style de vie des jeunes. Bien plus, ils doivent composer avec des jeunes en difficulté dont le comportement est parfois très différent de celui de leurs propres enfants. Ces parents n'ont généralement pas de formation professionnelle en relation d'aide, mais ils veulent être outillés quant à leur rôle d'appui et de soutien auprès de jeunes. C'est dans le vécu au quotidien que les parents d'accueil sentent le besoin d'être appuyés en ce qui regarde les relations amoureuses des jeunes, la contraception et la prévention des MTS-sida; ils s'adressent d'ailleurs fréquemment aux Centres jeunesse à ce sujet. De plus, comme les autres familles québécoises, les familles d'accueil ne sont pas non plus à l'abri des peurs et des sujets tabous, tels que la sexualité et les MTSsida.

C'est en considérant l'ensemble de ces facteurs que le programme a été conçu, élaboré et réalisé ; les participants ont manifesté une grande ouverture au dialogue et le désir sincère d'aider les jeunes. Le fait de les reconnaître aptes à jouer un rôle de prévention auprès des jeunes a renforcé positivement leur image comme parents.

\section{Une clientèle motivée}

Les parents d'accueil se sont montrés avides de connaissances à propos de la prévention des MTS-sida, du comportement des jeunes, de leurs valeurs, mais aussi désireux d'échanger entre eux et avec les intervenants sur les moyens d'aider les jeunes qui leur sont confiés. Être parents d'accueil représente une tâche difficile. Bien qu'elle soit souvent gratifiante, cette tâche est différente de celle d'assumer la responsabilité de ses propres enfants. Plusieurs participants ont souligné l'importance des échanges entre parents d'accueil, indépendamment du sujet abordé. En d'autres mots, 
ces parents ont un grand besoin de parler, de comparer leur façon d'agir auprès des jeunes. Ce besoin des parents d'accueil de se confronter avec d'autres qui vivent la même expérience qu'eux est une des explications du haut degré de satisfaction des parents relativement au programme.

L'effet «rapprochement parents d'accueil et jeunes » est un autre aspect à souligner. En effet, la sexualité est un sujet intime, et aborder cette question avec un jeune en famille d'accueil demande doigté et empathie en plus de présupposer une bonne communication entre les parties. Nous avions postulé qu'une telle communication positive existait entre les parents d'accueil et les jeunes. En cours de programme, nous avons demandé aux participants d'aborder, avec les jeunes, le sujet des MTS et du sida de la façon qui leur paraissait la plus appropriée. La tâche demandée s'est révélée à l'origine d'une communication parfois très intime: deux jeunes ont parlé pour la première fois avec le parent d'accueil de l'abus sexuel dont elles avaient été victimes antérieurement. Une jeune a déclaré à sa mère d'accueil «qu'elle avait rajeuni » depuis qu'elle suivait la formation, et le contact affectif s'est renforcé dès ce moment entre elles. La fille d'une mère d'accueil s'est étonnée joyeusement: "Pas toi maman qui me parles de condom!». La satisfaction évidente avec laquelle les parents d'accueil ont relaté le succès de la tâche demandée donne à penser que le programme a augmenté chez eux le sentiment de compétence parentale. Les résultats de la tâche paraissent avoir agi en spirale: 1) désir du parent d'aborder le sujet des MTS-sida avec le jeune; 2) étonnement et réceptivité chez le jeune ; 3 ) ouverture à la communication parent-jeune ; 4) confidences et 5) satisfaction chez le parent, renforcement de son sentiment de compétence.

\section{Une approche globale}

Le contenu du programme basé sur la vie affective des jeunes plutôt que sur l'acquisition des connaissances et des techniques de prévention des MTS-sida représente une de ses caractéristiques principales. Les jeunes, on le sait par les milieux de pratique, sont «fatigués» d'entendre parler des MTS et du sida; ils veulent qu'on leur parle de l'amour, des relations garçons-filles. Les parents d'accueil ont été sensibles à cette approche globale, comprenant que les activités sexuelles s'inscrivent dans une perspective plus large que celle de la seule génitalité. 


\section{Une approche interdisciplinaire}

et en partenariat avec la recherche

Le programme aborde trois dimensions: l'aspect psychosocial, l'aspect santé et les caractéristiques des jeunes en protection et en réadaptation. L'interdisciplinarité trouve ici un lieu d'exercice privilégié. Tant pour la conceptualisation que pour l'actualisation du programme, les intervenantes, de formations différentes, ont constamment interagi, se renforçant mutuellement et par la même occasion renfonçant la crédibilité de leurs messages. Les résultats sommatifs du projet montrent que les participants ont reconnu leur compétence et leur expertise et qu'ils ont particulièrement apprécié la connaissance immédiate qu'elles pouvaient avoir des jeunes confiés au Centre jeunesse de Québec.

Le partenariat avec des chercheurs externes de milieu universitaire a également ajouté à la crédibilité du programme, tant pour les intervenantes que pour l'établissement-hôte et les parents d'accueil. En effet, même si le processus de l'expérimentation et de l'évaluation avait été bien expliqué aux participants, la présence des chercheurs, en début et en fin de session, loin de les rebuter, leur a au contraire plu. En plus de présenter un intérêt sécurisant dans la démarche expérimentale d'accompagnement, la procédure convenue validait l'expérience d'apprivoisement mutuel.

\section{Une approche apparentée au "changement de type émergent"}

Sans être inspiré directement du modèle de "prévention par le changement émergent» (Schoonbroodt et Gélinas, 1996), le programme en possède un certain nombre de caractères. En effet, dans la conception du "changement émergent ",

[le] changement est émergent des interactions entre des acteurs dans un contexte donné. Il est contingent des intentions et des enjeux portés par les acteurs. [...] Cette perspective éducative de médiation se traduit par des approches fondées sur la conception de la gestion appropriative, traduction française d'empowerment.

(Schoonbroodt et Gélinas, 1996)

Le programme, tel que mis en ouvre au Centre jeunesse de Québec, a laissé une large place aux participants quant à l'expression de leurs besoins et quant au choix de leurs stratégies de prévention auprès des jeunes. Les intervenantes ont voulu tout au long du programme appuyer les capacités d'agir des participants. 


\section{CONCLUSION}

Trois leçons sont à tirer de l'expérimentation de ce programme pour la pratique professionnelle et la recherche en service social:

1) Faire confiance aux parents d'accueil

Ce sont eux-mêmes des parents, ouverts aux besoins des enfants des autres. Ce sont des personnes significatives auprès de jeunes qui leur sont confiés et leurs compétences comme parents pourraient être mises à contribution, non seulement dans le domaine de la prévention des MTS et du VIH-sida, mais dans toute autre problématique touchant la jeunesse. Ils constituent une richesse dans le champ de la pratique professionnelle en travail social.

2) Ne pas avoir peur des programmes interdisciplinaires d'intervention

Au Québec, les services de santé et les services sociaux ont longtemps été deux mondes parallèles (Berlinguet, 1996). Il est temps d'apprendre à travailler ensemble. C'est stimulant, efficace et "l'fun», diraient les intervenantes dans les milieux de pratique.

3) Approfondir le modèle de prévention par le changement émergent

Ce modèle est en effet à retenir, puisqu'il vise l'appropriation significative. La discipline du service social, basée sur la capacité des personnes à changer et sur le principe de l'autodétermination, a compris cela depuis longtemps. 


\section{Références bibliographiques}

BERLINGUET, M. (1996). "Recherche sur le sida», Les succès et les échecs de la recherche socio-médicale, Actes du colloque du Conseil québécois de la recherche sociale, tenu à Montréal le 13 mai 1996, Gouvernement du Québec. (À paraître)

GODIN, G. (1991). «L'éducation pour la santé : les fondements psychosociaux de la définition des messages éducatifs », Sciences sociales et santé, vol. IX, $\mathrm{n}^{\circ} 1$.

SCHOONBROODT, C. et A. GÉLINAS (1996). «La prévention par le changement émergent: apprendre à gérer les problèmes!", Éducation santé, 108.

\section{Notes}

1. Guide d'animation du programme de formation «Parents d'accueil et prévention des MTS et du VIH-sida chez les adolescentes et les adolescents à risque », MSSS 1996. Le guide est disponible dans les Régies régionales de la santé et des services sociaux.

2. «Le rôle éducatif des parents d'accueil en matière de prévention des MTS et du sida », Symposium sur la famille, Trois-Rivières, mai 1995. «La prévention des MTS/sida auprès d'adolescents placés en famille d'accueil : résultats d'un programme de formation auprès des parents ", Colloque de l'Ordre professionnel des travailleurs sociaux du Québec, Sherbrooke, 31 mai 1996. Le projet a été présenté avec la participation de deux parents d'accueil.

3. «La santé et ses déterminants », Forum national sur la santé, septembre 1996, p. 43.

4. Colloque de l'Association des Centres jeunesse du Québec, Laval, 18-19 octobre 1996.

5. Nicole Gagné, travailleuse sociale; Pierrette Beaupré, agente de relations humaines; Josée Brousseau, éducatrice; Céline Brochu et Rachel Lavigueur, infirmières. 